\title{
Numerical Simulation Study on Large Kitchen Oil Fume Purification Device
}

\author{
Ning $\mathrm{Li}^{\mathrm{a}}$, Weilesu Wang ${ }^{\mathrm{b}}$, Baoyin $\mathrm{Ma}^{\mathrm{c}}$, Hongyin $\mathrm{Yu}^{\mathrm{d}}$, Jiaming Kang ${ }^{\mathrm{e}}$, Wenting \\ Sun ${ }^{\mathrm{f}}$, Yinglai Cai ${ }^{\mathrm{g}}$, Xiang $\mathrm{Gou}^{\mathrm{h}}$
}

School of Energy and Environmental Engineering, Hebei University of Technology, Tianjin, 300401, China

aemail:In120691@126.com, bemail: 1697567753@qq.com, cemail: 2434542871@qq.com, demail: yhy-1993@163.com, eemail: 1071431062@qq.com, ${ }^{\mathrm{f} e m a i l: 515979477 @ q q . c o m, ~}{ }^{\mathrm{g} e m a i l:}$

caiyinglai@qq.com, ${ }^{\mathrm{h}}$ Corresponding author email: gouxiang@sina.com

Keywords: Numerical Simulation; Fume Purification; Velocity Distribution; Pressure Distribution; Removal Rate

Abstract. This paper focuses on a large kitchen fume gas purification device newly designed by numerical simulation using FLUENT software. The velocity distribution and pressure distribution within the whole device are simulated. Particle trajectories of different droplet diameters $(5 \mu \mathrm{m}, 8 \mu \mathrm{m}$, $11 \mu \mathrm{m}$, and $15 \mu \mathrm{m}$ ) are also simulated respectively and the device removal rate of different diameter droplets were $60.86 \%, 76.34 \%, 99.89 \%$ and $100 \%$ respectively. It is found that the novel device has very good ability to remove particles in the fume gas.

\section{Introduction}

With the rapid development of our country's economy, the catering industry is constantly expanding, the environmental pollution in the restaurant industry and residents kitchen lampblack pollution problems brought by the increasingly serious. Without treated fumes discharges into the air, and pollutes the environment and endangers human. Currently handles cooking fumes, the major domestic use inertial separators, electrostatic deposition method, liquid scrubber, cellular deposition method, compound purification method and so on[1], it is mainly used in foreign oxidation incineration, catalytic oxidation technology. Jianli Wang prepared a low ignition temperature and high selectivity of the catalyst, and the catalyst had a lower catalytic reaction temperature and better catalytic efficiency than common catalyst[2]. Ji Yang studied low temperature catalyst[3], etc. However, many methods have higher cost or lower efficiency, so the development of environmental-friendly lampblack purification technology is currently a major demand to solve the problem of environmental pollution. At present, Yaqian Liu researched "cyclone off the development and optimization of exhaust hood", which introduces the cyclone off the hood and exhaust developed a liquid cyclone in its internal main gas separation equipment fume Optimal design[4]. Cheng Kai studied the "cyclone-type fume purification Principle Analysis and Numerical Simulation", mainly for new cyclone fume purifier dynamic analysis, internal flow field simulation, to obtain the forces and means of purification process velocity field, pressure field, thereby affecting the purifying effect of the design parameters and operating conditions were analyzed and optimized for best results [5]. New smoke lampblack machine is introduced in this paper on their research conducted on the basis of improving, and increases the inertia separation method. The oil droplets in flue gas are collected mainly through the inertia separation flue and cyclone separator. It has the advantage of small volume, light weight, small occupied place, large processing capacity per unit area, high oil removal efficiency. The disadvantage is that the oil viscosity is high and it is easy to stick on the wall, so it needs regular cleaning.

This article mainly uses GAMBIT software to build a model, and then imports it to FLUENT for simulate calculation. The velocity distribution and pressure distribution within the whole device are simulated. Particle trajectories are also simulated for the four different droplet diameters $(5 \mu \mathrm{m}, 8 \mu \mathrm{m}$, $11 \mu \mathrm{m}$, and $15 \mu \mathrm{m}$ ) respectively. Geometric model of the device is shown in Fig. 1. 


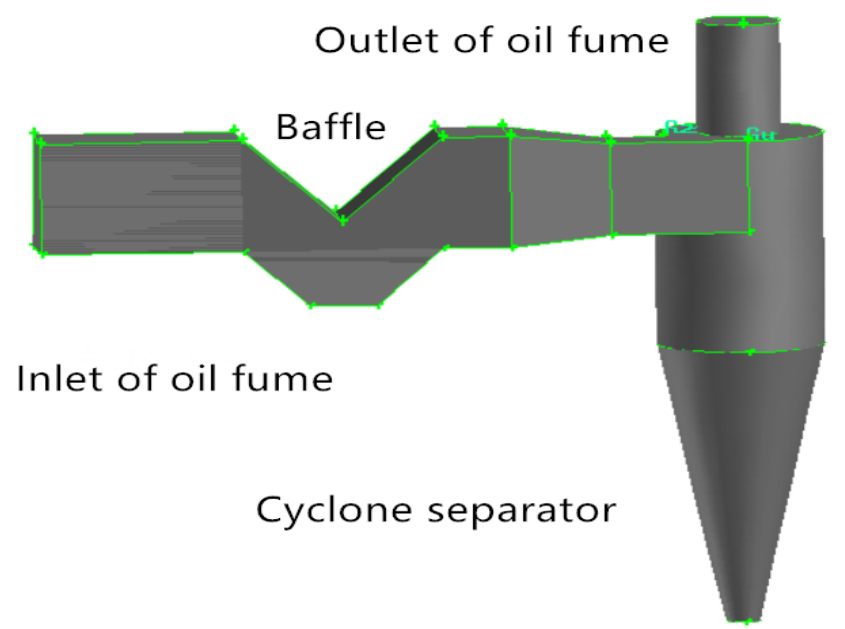

Fig. 1. Geometric model of the device

\section{The theory of simulation calculation}

(1) Mass conservation equation

Any flow must satisfy the law of conservation of mass, the law can be expressed that the increasing mass flowing micro element per unit time is equal to the net mass inflow of the micro body at the same time interval. The mass conservation equation:

$$
\frac{\partial \rho}{\partial \mathrm{t}}+\nabla \bullet(\rho V)=S_{\mathrm{m}}
$$

Where $\rho$ is density and $t$ is time and $V$ is the velocity vector, and $S_{m}$, the source phase, which is added to the mass of the continuous phase. There is a general form of mass conservation equation, which is suitable for the compression flow and incompressible flow.

(2) Momentum conservation equation

The momentum conservation equation is the basic law that must work on any flow problem, and its essence is Newton's second law. The law can be expressed as: the rate of change of fluid momentum with time in micro body is equal to sum of all kinds of forces on the element. In the inertial coordinate the momentum conservation equations can be obtained:

$$
\frac{\partial(\rho V)}{\partial \mathrm{t}}+\nabla \bullet(\rho V V)=-\nabla \mathrm{p}+\nabla \bullet(\tau)+\rho \mathrm{g}+F
$$

Where $\rho$ is the fluid static pressure; $\mathrm{G}$ and $\mathrm{F}$ represent gravity volume force on the micro element and other external volume force respectively; $\tau$ is viscous stress tensor on the surface of element, which originates the molecular viscosity. The equation is established for any type of fluid [6].

(3) Set of simulation conditions

1. Using two equation turbulence model (RNG $\kappa-\varepsilon)$;

2. Discrete phase stochastic trajectory model. The motion trajectory of oil droplets in the Lagrange coordinate system is studied by numerical simulation;

3. Most of the oil droplets diameter range is $1-20 \mu \mathrm{m}$;

4. The uniform distribution is used to study the droplet diameter;

5. Import speed is $20 \mathrm{~m} / \mathrm{s}$.

\section{Results and discussion}

1. Velocity analysis

Internal velocity distribution of the device is showed in Fig. 2. It can be seen that velocity obviously decreases in the baffle plate; in the cyclone separator, velocity decreases gradually from the top down and achieves the minimum in the bottom. Oil droplets easily gather where there is a marked decline in velocity so that the oil droplets could be collected in these places. It can be seen 
that the back of the baffle plate, the lower part of the baffle and the bottom of the cyclone separator are easier to collect the oil droplets.

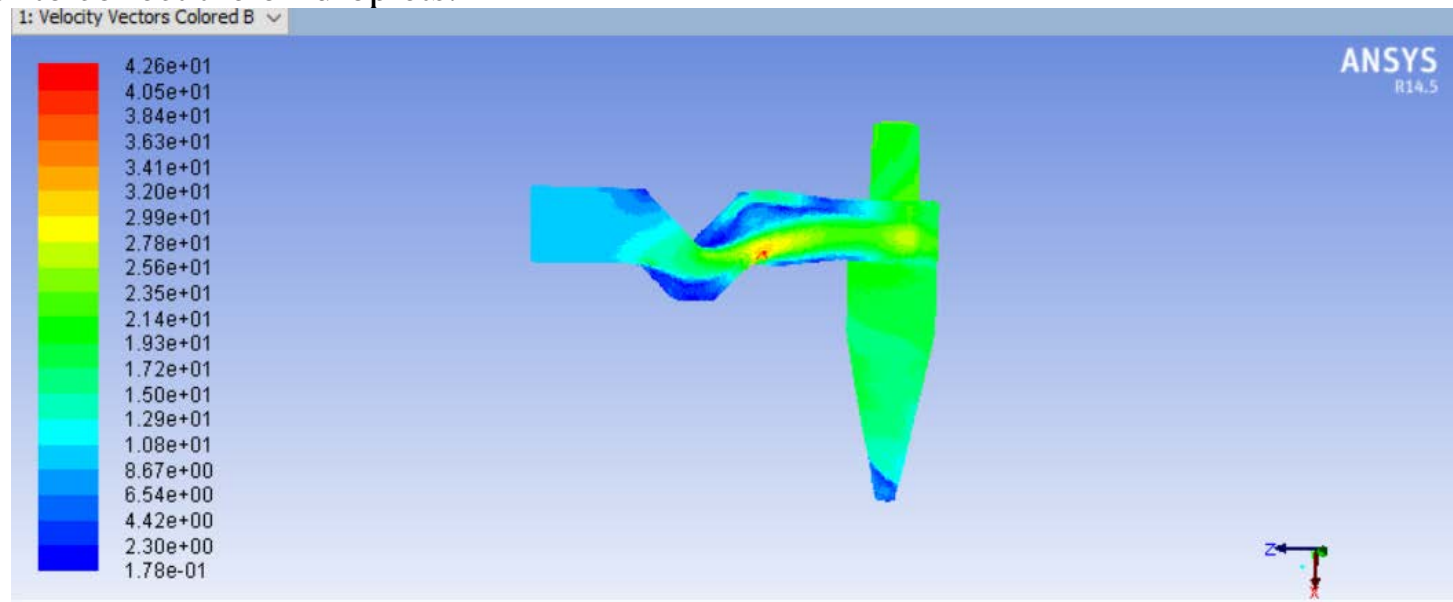

Fig. 2. Velocity distribution of device

$X=300$ and $X=500$ are cross sections of the cyclone separator, which is used to analyze tangential velocity. As shown in Fig. 3, from the figure it can be seen that the rotary airflow of cyclone separator is not the axial symmetry, which explains that the air flow in the cyclone is not strictly upward along the axis, there will be a swing, which confirms the import of the cyclone separator is located in the side rather than the middle.

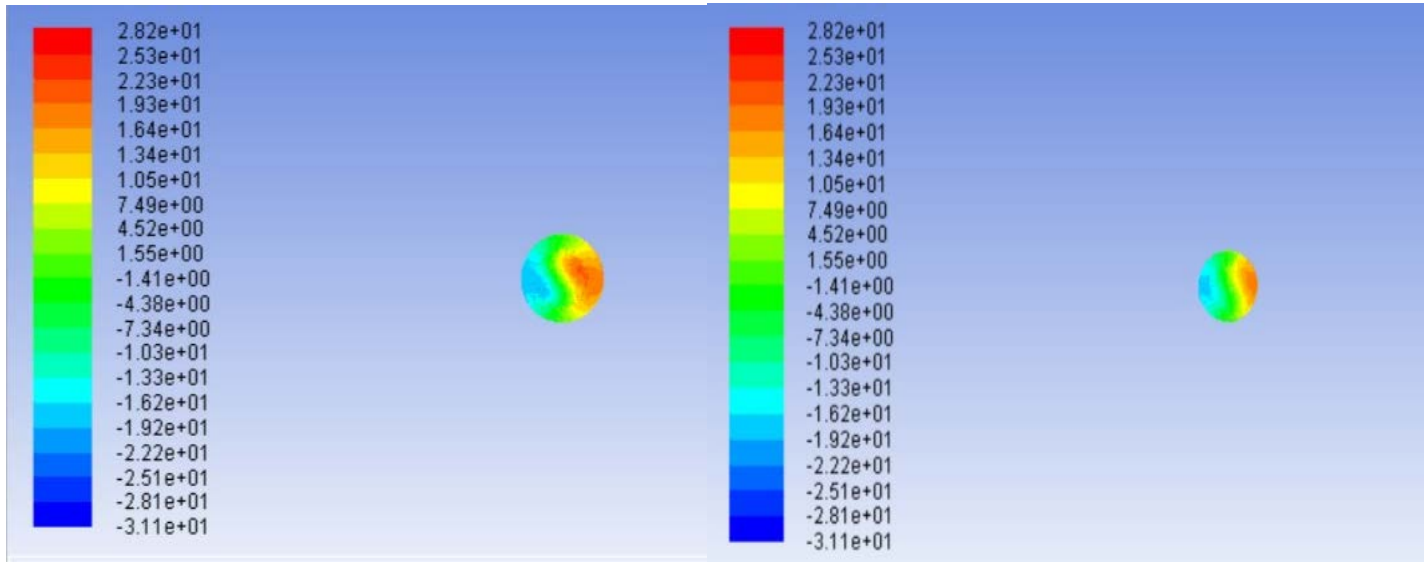

(a)Velocity tangential distribution of $\mathrm{X}=300$

(b)Velocity tangential distribution of $\mathrm{X}=500$

Fig. 3. Tangential velocity distribution

\section{Pressure analysis}

The pressure field distribution of cyclone separator is showed in Fig. 4, in the same horizontal section, the pressure decreases gradually from the wall to the center and size and basically assumes axial symmetry. At the connection of the entrance and the wall, the pressure has a great fluctuation which is not obvious along the wall. When there is a vortex in the center of the cylinder, there will be a negative pressure.

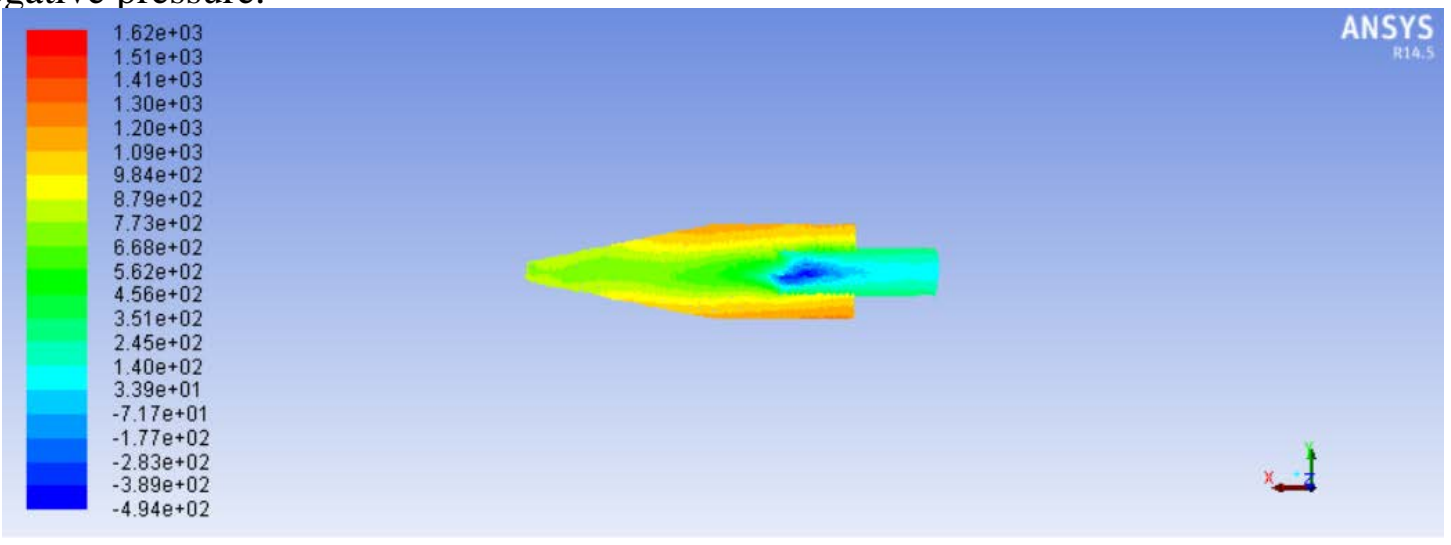

Fig. 4. Pressure distribution 


\section{Oil droplets trajectories analysis}

Since the diameter of most of the oil droplets is at $1-20 \mu \mathrm{m}$, the main the droplet diameters of simulation are $5 \mu \mathrm{m}, 8 \mu \mathrm{m}, 11 \mu \mathrm{m}$, and $15 \mu \mathrm{m}$. The trajectory of oil droplets of different diameters are showed in Fig. 5.

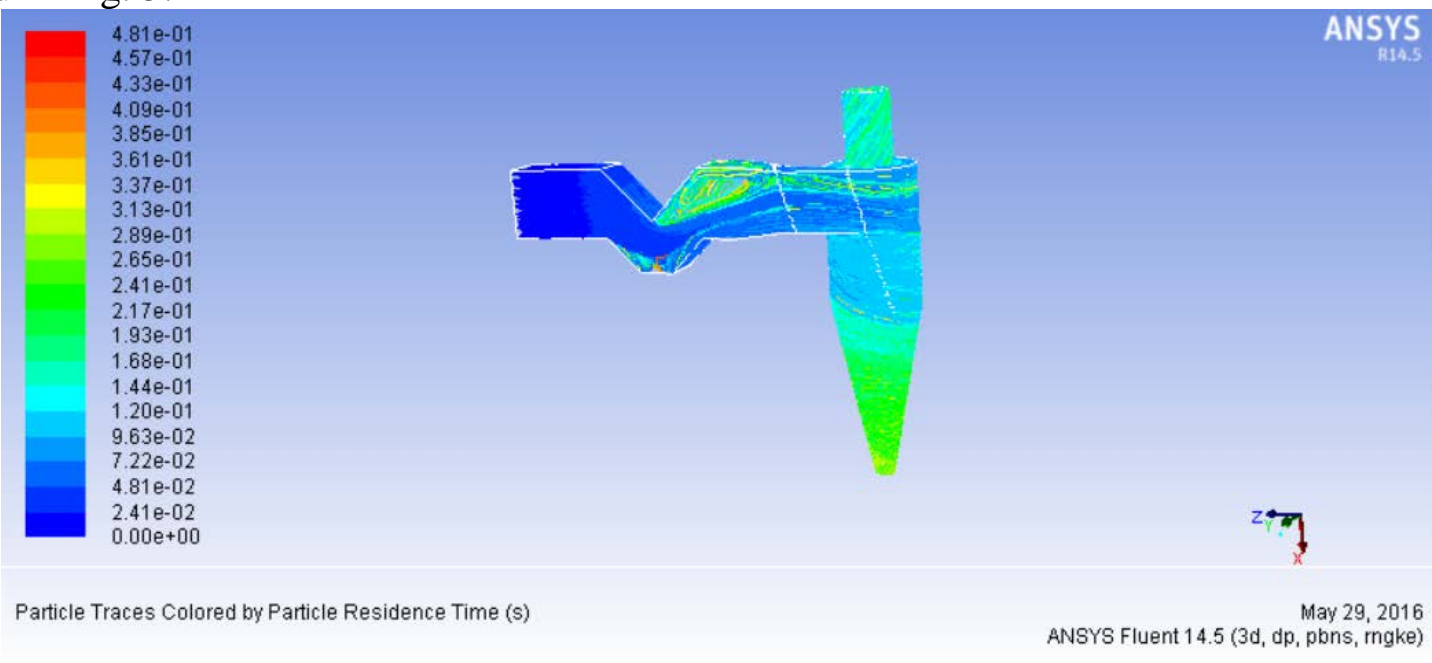

DPM Iteration ....

number tracked $=930$, escaped $=364$, aborted $=0$, trapped $=0$, evaporated $=0$, incomplete $=566$, incon

(a) Particle trajectory of oil droplet diameter of $5 \mu \mathrm{m}$

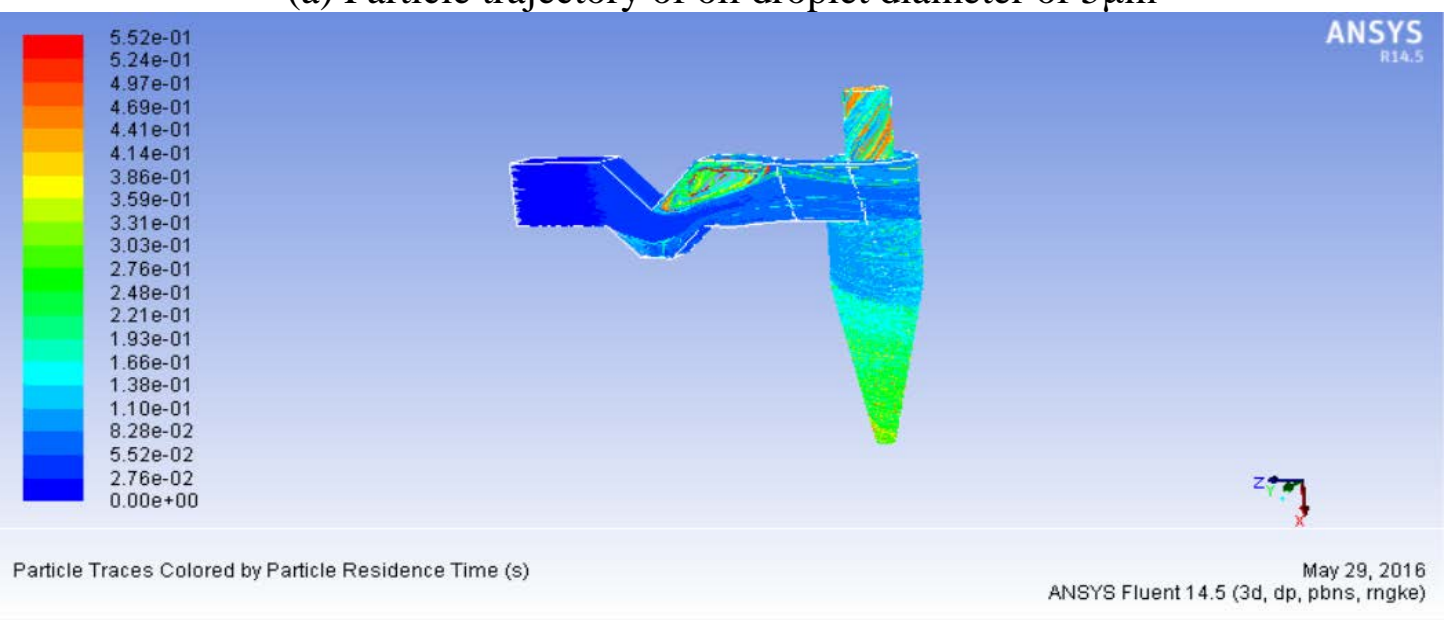

DPM Iteration ....

number tracked $=930$, escaped $=220$, aborted $=0$, trapped $=0$, evaporated $=0$, incomplete $=710$, incon

(b) Particle trajectory of oil droplet diameter of $8 \mu \mathrm{m}$

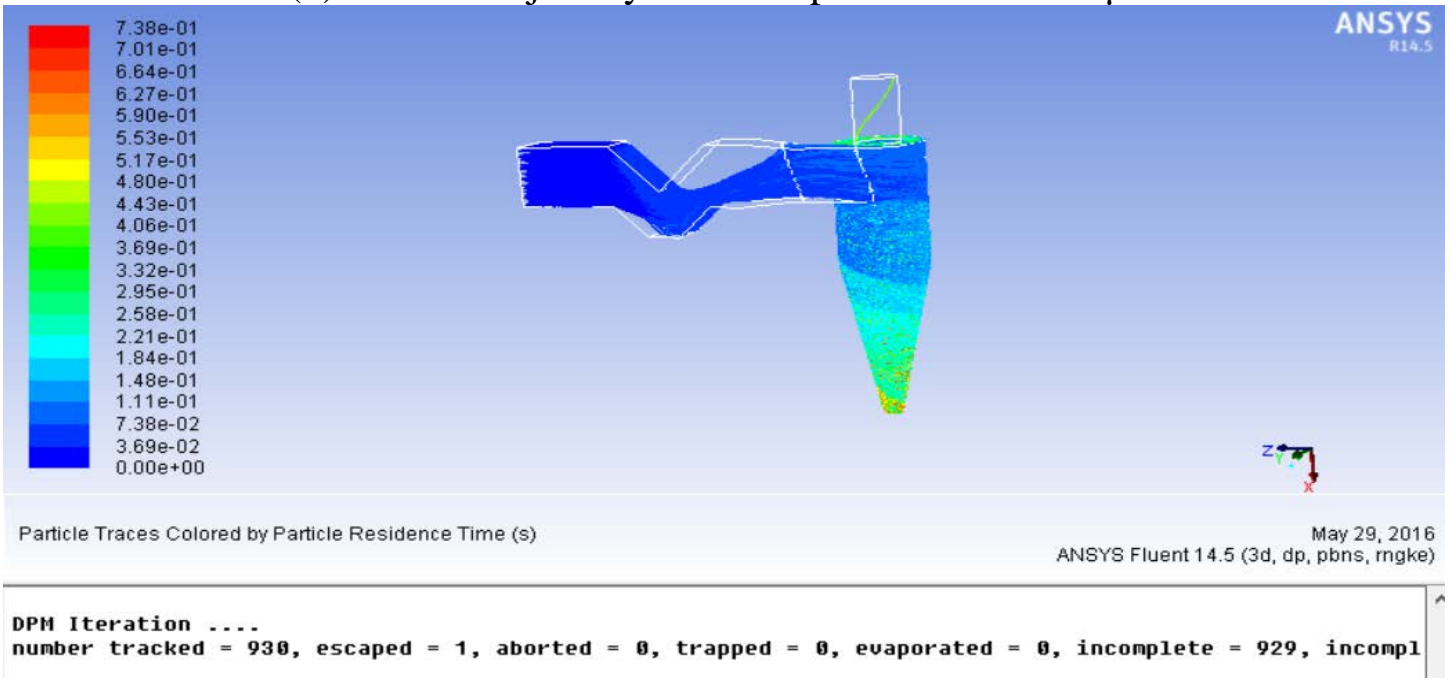

(c) Particle trajectory of oil droplet diameter of $11 \mu \mathrm{m}$ 


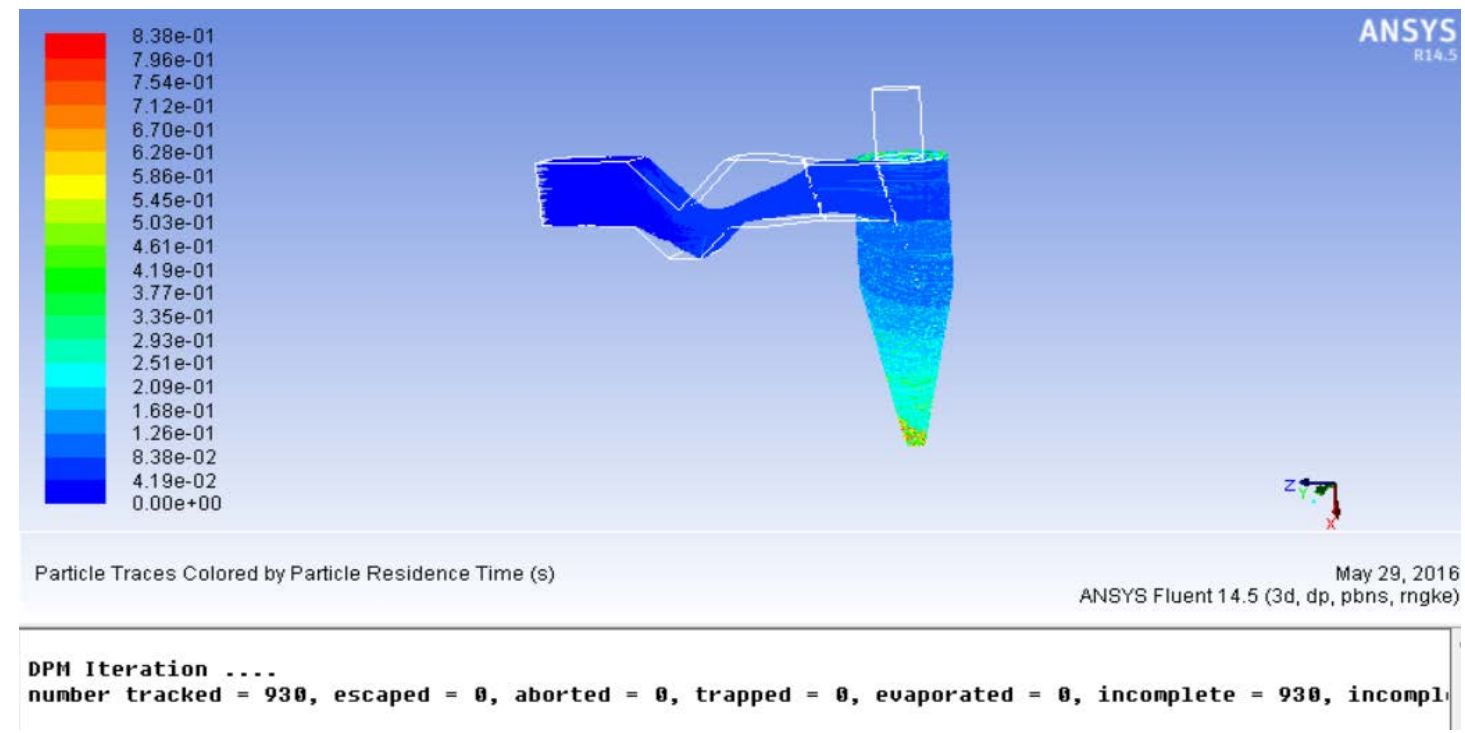

(d) Particle trajectory of oil droplet diameter of $15 \mu \mathrm{m}$

Fig. 5. The distribution of oil droplet trajectory

From the Fig. 5 it can be seen that the following nature of particles which diameter is $5 \mu \mathrm{m}$ and $8 \mu \mathrm{m}$ is very good, with rotating airflow downward movement, part of the oil droplets arrive at the bottom of the cone part and spiral up with internal rise, get into the exhaust pipe. Part of the oil droplets spiral before they arrive at the bottom, and rise by rotating gas flow, and directly get into the exhaust pipe.

Larger centrifugal force of oil droplets of $11 \mu \mathrm{m}$ and $15 \mu \mathrm{m}$ and more prominent gravity make oil droplets be easily left to cyclone wall and slide rotatably to the bottom of the ash bucket along the wall and separate in the end. The number of rotations is far less than the oil droplets. It can be seen that large particles are more likely to be tracked by the original motion.

The removal rates of oil droplets of $5 \mu \mathrm{m}, 8 \mu \mathrm{m}, 11 \mu \mathrm{m}$ and $15 \mu \mathrm{m}$ are $60.86 \%, 76.34 \%, 99.89 \%$, $100 \%$ respectively. It is shown that the large droplets are more likely to be captured, the removal rate is higher.

\section{Summary}

A new large kitchen oil smoke purifying device is proposed and simulated using FLUENT software. The device's internal velocity and pressure distribution can be clearly provided, which can make a reasonable improvement of the internal structure of the device. Four different droplet diameters $(5 \mu \mathrm{m}, 8 \mu \mathrm{m}, 11 \mu \mathrm{m}$, and $15 \mu \mathrm{m})$ of particle trajectory are simulated respectively. It is shown that bigger droplet diameter is easier to be caught.

\section{Acknowledgement}

In this paper, the research was sponsored by College Students Innovation and Entrepreneurship Training Program (Project No. DC201510080037) and Research Funds for College Students of Modern Marine Chemical Engineering Technology Synergy Innovation Center of Hebei Province (Grant No. HYHG201513).

\section{References}

[1] Mingjun Pang, Dingbiao Li, Mao Sun. Oil fume purifying method and purifying mechanism [J]. Journal of Nanchang College of Water Conservancy and Hydroelectric Power. (In Chinese)

[2] Jianli Wang. Removal of volatile organic gas catalytic combustion monolithic catalyst [D]. Sichuan University, 2007. (In Chinese)

[3] Ji Yang, Jinping Jia, Yaling Wang,Weisong You.Treatment of cooking oil fume by low 
temperature catalysis [J]. Applied Catalysis B: Environmental, Volume 58, Issues 1-2, 8 June 2005, Pages 123-131.

[4] Yaqian Liu. Development and optimum design of the cyclone type exhaust hood [D]. Anhui University of Science and Technology, 2012. (In Chinese)

[5] Kai Cheng, Tao Zeng, Zhongbin Liu, Shaobei Liu, Wei Yang. Principle analysis and numerical simulation of cyclone separation type fume purifier [J]. Filtration and separation, 2012, 01: 11-13+27. (In Chinese)

[6] Yong Yu. FLUENT introductory and advanced tutorials [M]. Beijing: Beijing Institute of Techenology Press, 2008. 9. (In Chinese) 